\title{
Atendimento humanizado em harmonização orofacial: revisão integrativa da
}

\section{literatura}

\author{
Humanized care in orofacial harmonization: integrative literature review \\ Atención humanizada en la armonización orofacial: revisión de la literatura integradora
}

Recebido: 12/10/2021 | Revisado: 19/10/2021 | Aceito: 26/10/2021 | Publicado: 30/10/2021

\author{
Alessandro Ítalo Cruz \\ ORCID: https://orcid.org/0000-0003-4679-7302 \\ Centro Universitário Tiradentes, Brasil \\ E-mail: aleitalocruz@gmail.com \\ Ana Karlla Santos Rocha \\ ORCID: https://orcid.org/0000-0002-5746-8881 \\ Centro Universitário Tiradentes, Brasil \\ E-mail: anakarllinharocha@hotmail.com \\ Bruna Rafaella Ramos Melo \\ ORCID: https://orcid.org/0000-0003-4186-4270 \\ Centro Universitário Tiradentes, Brasil \\ E-mail: brrm.bruna@gmail.com \\ Joedy Maria Costa Santa Rosa Lima \\ ORCID: https://orcid.org/0000-0001-8486-0128 \\ Centro Universitário Tiradentes, Brasil \\ E-mail: joedysantarosa@ hotmail.com \\ Palmyra Catarina Costa Santa Rosa Lima \\ ORCID: https://orcid.org/0000-0002-7017-524X \\ Centro Universitário Tiradentes, Brasil \\ E-mail: drapalmyrasantarosa@gmail.com
}

\begin{abstract}
Resumo
A humanização vem sendo prática fundamental para o novo perfil do cirurgião-dentista. O paciente é um ser completo, que almeja por profissionais capazes tecnicamente, e ao mesmo tempo desfrutar de experiências baseadas no aspecto humano. Objetivo: contribuir para discussão sobre a atuação do cirurgião-dentista no âmbito da HOF, com ênfase na humanização. Métodos: Trata-se de uma revisão integrativa da literatura, de caráter exploratório, com abordagem qualitativa, segundo Mendes (2008), onde foi utilizada a base de dados: Scielo. Para o desenvolvimento da pesquisa, foram aplicados os descritores: "Odontologia", "Humanização", "Orofacial". Resultados: Os números de intervenções estéticas na Odontologia têm crescido cada vez mais. Essa busca está ligada ao desejo de melhorar o bem estar biopsicossocial. Para entender a crescente busca pela harmonização orofacial, é preciso observar como a odontologia vem tratando o paciente: indo além do que é padronizado. Nessa abordagem estão implícitos: ouvir, acolher; esclarecer; sanar dúvidas, permitindo que o paciente participe do seu plano de tratamento. Conclusão: Podese notar que a humanização vem sendo exaltada como algo essencial na Odontologia, em decorrência da valorização do rosto e do sorriso na vida das pessoas.
\end{abstract}

Palavras-chave: Odontologia; Humanização; Orofacial.

\begin{abstract}
Humanization has become a fundamental practice for the new profile of the dental surgeon. The patient is a complete being, who longs for technically capable professionals, and at the same time enjoy experiences based on the human aspect. Objective: To contribute to the discussion about the performance of the dental surgeon in the context of the HFOO, with emphasis on humanization. Methods: Methods: This is an integrative literature review, exploratory in nature, with a qualitative approach, according to Mendes (2008), which used the database: Scielo. For the development of the research, the descriptors were applied: "Dentistry", "Humanization", "Orofacial". Results: The numbers of aesthetic interventions in dentistry have been growing more and more. This search is linked to the desire to improve biopsychosocial well-being. To understand the growing search for orofacial harmonization, it is necessary to observe how dentistry has been treating the patient: going beyond what is standardized. Implicit in this approach are: listening, welcoming, clarifying, answering questions, and allowing the patient to participate in his/her treatment plan. Conclusion: It can be noted that humanization has been exalted as something essential in dentistry, due to the appreciation of the face and the smile in people's lives.
\end{abstract}

Keywords: Dentistry; Humanization; Orofacial. 


\begin{abstract}
Resumen
La humanización se ha convertido en una práctica fundamental para el nuevo perfil del cirujano dental. El paciente es un ser completo, que anhela contar con profesionales técnicamente capacitados y, al mismo tiempo, disfrutar de experiencias basadas en el aspecto humano. Objetivo: contribuir a la discusión sobre la actuación del cirujano-dentista en el ámbito del HOF, con énfasis en la humanización. Métodos: Se trata de una revisión bibliográfica integradora, de carácter exploratorio, con enfoque cualitativo, según Mendes (2008), que utilizó la base de datos: Scielo. Para el desarrollo de la investigación se aplicaron los descriptores: "Odontología", "Humanización", "Orofacial". Resultados: El número de intervenciones estéticas en odontología ha ido creciendo cada vez más. Esta búsqueda está vinculada al deseo de mejorar el bienestar biopsicosocial. Para entender la creciente búsqueda de la armonización orofacial, es necesario observar cómo la odontología ha estado tratando a los pacientes: yendo más allá de lo estandarizado. Este enfoque lleva implícito: escuchar, acoger, aclarar, responder a las preguntas, permitir que los pacientes participen en su plan de tratamiento. Conclusión: Se puede observar que la humanización se ha exaltado como algo esencial en la odontología, debido a la valoración del rostro y la sonrisa en la vida de las personas.
\end{abstract}

Palabras clave: Odontología; Humanización; Orofacial.

\title{
1. Introdução
}

Humanizar é o mesmo que atribuir essência humana a algo; tornar um ofício em algo humanizado. Assim, compreende-se que o ato de humanizar algo é inerente e totalmente intrínseco à ação humana. Não se pode separar uma pessoa em partes como: dentes, ossos, músculos, sistemas, etc (Kessamiguiemon et al., 2017). A humanização vem sendo preconizada na legislação vigente como essencial no processo de formação do cirurgião-dentista. Sendo, portanto, considerada como prática fundamental para a construção do novo perfil esperado para um cirurgião-dentista. (Canalli et al., 2011). Deve-se ter entendimento que o paciente é um ser completo, que ao momento em que procura ajuda de um profissional da saúde, da Odontologia, traz consigo toda a sua bagagem emocional, cultural, que lhes confere medos, frustrações, dúvidas, expectativas e esperanças individuais (Kessamiguiemon et al., 2017).

O profissional que se encarrega de prestar esses cuidados deve ouvir o paciente - não apenas seus sintomas e suas queixas físicas; mas, acolher este paciente com atenção e interesse sincero em seu estado, informá-lo sobre os procedimentos antes mesmo que sejam executados, bem como receber suas sugestões e sanar suas dúvidas (Kessamiguiemon et al., 2017). O cuidado humano precisa estar apoiado em atitudes éticas, que são ensinadas durante todo o processo de formação profissional (Rezende et al., 2015). As pessoas almejam por profissionais capazes tecnicamente, ao mesmo tempo em que anseiam desfrutar de experiências baseadas no aspecto humano, onde sejam notadas em todo seu aspecto social (Guerra et., al. 2015); (Kessamiguiemon et al., 2017).

O cirurgião-dentista que demonstra atitude empática durante o atendimento odontológico, mostrando respeito às queixas e aos sentimentos do paciente, que elabora uma explicação clara de cada procedimento, poderá certamente diminuir e até suprir a ansiedade de seus pacientes, já que sentimentos de confiança, segurança, tranquilidade e serenidade são estimulados durante as consultas (Rezende et al., 2016). Embora a humanização já esteja englobada em todas as áreas da saúde, a prática ainda é pauta recente no que se refere às áreas que abrangem mais fortemente a estética, como é o caso da Harmonização Orofacial Garbin et al., (2019) frisou o quanto as alterações estéticas faciais e a insatisfação da autoimagem têm repercussões negativas sobre a saúde emocional e a vida do indivíduo. Para algumas dessas pessoas, cirurgias e procedimentos estéticos são o caminho ideal para melhorar a imagem social e aumentar a autoestima (Borba et al.,2018).

O conceito do que é belo ao longo da história, sempre foi entendido como uma questão subjetiva e individual, baseado em valores culturais, raciais, étnicos, assim como pela escuta da opinião familiar, popular e contextualização atual do que está na moda e exposto nas mídias (Garbin et al., 2019). Atualmente, as pessoas buscam reconhecimento nos demais, através da sua imagem. Essa procura por procedimentos estéticos está vigorosamente ligada à vontade de melhorar a aparência e a autoestima, aprimorando assim a qualidade de vida e o bem estar psicológico (Rezende et al., 2016). Por se tratar do 
segmento do corpo mais representativo e valorizado pelo ser humano, a face, normalmente, é onde se concentram os esforços para promoção e conservação de estética (Moura, 2021).

A face humana pode ser classificada em três tipos: dolicocefálica (longa e estreita) braquicefálica (curta e com largura aumentada) e mesocefálica (tipo intermediário) (Júnior et al., 2018). Por ser uma estrutura complexa composta por tecidos e órgãos que estão totalmente envolvidos um no outro, em diferentes camadas, profundidades e regiões, por isso, é indispensável ao profissional que atua na Harmonização orofacial, a realização de um estudo detalhado (Schmidt, 2021). A análise facial é utilizada a fim de avaliar características, através de fotografias e exames de imagem, definindo proporções, volume, aparência, simetria e deformidade e pode ser dividida em análise frontal e análise do perfil. Além disso, através dos fundamentos da análise facial, baseado em uma referência do padrão do biotipo brasileiro, pode-se diagnosticar uma desarmonia de ordem estética, dentária ou de alteração anatômica que poderá ser corrigida (Júnior et al., 2018).

Para avaliar a simetria e o equilíbrio da face, é muito usada a prática de dividi-la horizontalmente em três terços. O terço superior se estende da inserção do cabelo à glabela, o terço médio da glabela à região subnasal, e o terço inferior da região subnasal ao mento (Machado, 2020). Na análise do perfil é possível identificar três tipos de perfis no paciente: côncavo, convexo e reto. Na análise do sorriso são observadas, a forma do sorriso, às margens gengivais, as bordas incisais dos incisivos, como também a posição e tamanho dos dentes. Um sorriso considerado agradável e com características de jovialidade pode ser definido como o que expõe completamente os dentes superiores, podendo apresentar uma exposição gengival de $1 \mathrm{~mm}$ a $3 \mathrm{~mm}$ (Júnior et al., 2018). Os avanços tecnológicos da odontologia no Brasil, junto ao aumento da busca por procedimentos estéticos, vêm permitindo que os dentistas melhorem a harmonia estética da face, aplicando técnicas orofaciais que complementam um belo sorriso e melhoram a estética facial como um todo (Machado, 2020).

O objetivo deste estudo é contribuir para discussão a respeito da importância da atuação do cirurgião-dentista no âmbito da harmonização orofacial, com ênfase na humanização, que influenciará diretamente no fator emocional, na autoestima e satisfação do paciente, quanto ao resultado dos procedimentos.

\section{Metodologia}

Trata-se de uma revisão integrativa da literatura Trata-se de uma revisão integrativa da literatura, de caráter exploratório, com abordagem qualitativa, cujo objetivo é descrever acerca do atendimento humanizado em harmonização orofacial, permitindo ao pesquisador uma investigação minuciosa sobre a problemática em questão ao realizar buscas, análises críticas e sínteses das comprovações que estão disponíveis acerca do tema abordado, com a finalidade de sintetizar o conhecimento e, também, ressaltar resultados de estudos relevantes já publicados (Mendes, Silveira \& Galvão, 2008). Para elaborar essa produção científica foram seguidas seis etapas: definição da pergunta norteadora, amostragem na literatura, extração de dados, análise crítica dos estudos incluídos, interpretação e síntese dos resultados e apresentação da revisão integrativa (Souza, Silva \& Carvalho, 2010).

Inicialmente o objetivo foi responder à pergunta norteadora do presente estudo que é: "Como proceder um atendimento humanizado em harmonização orofacial?”. Em seguida realizou-se a busca de artigos que correspondem entre os anos de 2010 até maio de 2021, no idioma inglês e português. Para a seleção desses foram utilizados os seguintes descritores: "Odontologia", "Humanização", "Orofacial”. Esta seleção ocorreu através da plataforma Descritores em Ciências da Saúde (DeCS). A base de pesquisas abordada foi Scientific Eletronic Library Online (SciELO). Primeiro, pesquisaram- se descritores de modo individual e em seguida, foram feitas diversas buscas com cruzamentos entre eles utilizando o operador booleano "AND". Os artigos selecionados passaram por critérios de exclusão e inclusão. Entre os critérios de inclusão estão a análise do 
ano de publicação, artigo original e publicado na íntegra que respondessem à questão norteadora, idiomas de português e inglês. Após a análise e seguindo esses critérios não foram encontrados artigos, como mostra no Quadro 1.

Quadro 1: Publicações encontradas entre os anos de 2010 a 2021 segundo a base de dados SciELO.

\begin{tabular}{|l|l|l|l|l|}
\hline Descritor & Total de publições & Publicações filtradas & $\begin{array}{l}\text { Após } \\
\text { leitura } \\
\text { título }\end{array}$ & $\begin{array}{l}\text { Após a leitura do } \\
\text { resumo }\end{array}$ \\
\hline Odontologia & 3.338 & 689 & 0 & 0 \\
\hline Humanização & 1.294 & 751 & 4 & 0 \\
\hline Orofacial adontologia and & 587 & 216 & 0 & 0 \\
\hline $\begin{array}{l}\text { Odonãano } \\
\text { Humanizaço and }\end{array}$ & 54 & 5 & 0 & 0 \\
\hline $\begin{array}{l}\text { Odontologia } \\
\text { Orofacial }\end{array}$ & 19 & 0 & 0 \\
\hline $\begin{array}{l}\text { Humanização and } \\
\text { Ororfacial }\end{array}$ & 1 & 1 & 0 & 0 \\
\hline $\begin{array}{l}\text { Odontologia and } \\
\text { Humanização and } \\
\text { Orofacial }\end{array}$ & 0 & 0 & 0 & 0 \\
\hline
\end{tabular}

Fonte: Autores.

É possível observar no Quadro 1 que após realizar a busca pelos descritores: "Odontologia", "Humanização", “Orofacial” e seus cruzamentos foram encontradas 5.285 (cinco mil duzentos e setenta e cinco) produções científicas. Após aplicar o filtro com os critérios previamente estabelecidos de inclusão e exclusão foram 1.681 (mil seiscentos e oitenta e um). Em seguida, os títulos dos artigos foram lidos e 4 (quatro) produções literárias foram escolhidas. Por fim, os resumos foram lidos e 0 (zero) artigos atenderam e responderam a pergunta norteadora dessa produção.

\section{Discussão}

Nesta Revisão Integrativa não foram analisados artigos científicos selecionados de acordo com os critérios de inclusão estabelecidos. O tema humanização na odontologia é amplamente discutido, em vários aspectos, no entanto, não foram encontrados artigos que abordam a humanização no campo da Harmonização Orofacial. Além do mais, dentre os artigos selecionados, nota-se que a busca por uma odontologia mais humanizada está cada vez mais constante, exigindo novas descobertas e ampliação de possibilidades disponíveis.

Importantes mudanças de valores na sociedade aconteceram no final do século XX, o homo politicus deu espaço ao homo estheticus e afetou o comportamento dos indivíduos em relação a sua beleza (Strehlau et al., 2015). Os números de intervenções estéticas têm crescido cada vez mais, milhões de procedimentos cirúrgicos e não cirúrgicos são realizados no mundo, a fim de atender a padrões estéticos (Strehlau et al., 2015). De acordo com dados da Sociedade Brasileira de Cirurgia Plástica (SBCP, 2021), os procedimentos estéticos não cirúrgicos, tiveram um aumento de 32,5\%, entre o período de 2014 à 2018, no Brasil. A definição de estética, inicialmente, foi introduzida por Aristóteles, que relatou que uma pessoa podia se ver mais, ou menos agradável aos olhos de outras pessoas (Machado, 2020). Conceitualmente, estética é a apreciação da beleza, ou a combinação de qualidades que proporcionam intenso prazer aos sentidos, às faculdades intelectuais e morais (Reis et al., 2011). Este conceito, assim como os conceitos de beleza também mudaram ao longo do tempo e possuem variações quando são observados em diferentes raças e etnias, estando sujeitos a parâmetros socioculturais e conceitos atuais de moda (Machado, 2020). 
Segundo a Organização Mundial da Saúde (OMS), saúde é um estado completo de bem-estar físico, mental e social e não apenas a ausência de doenças (Machado, 2020). Nesse sentido a odontologia tem sido uma grande aliada, não apenas no restabelecimento da função e do bem-estar, mas, principalmente, na saúde do paciente como um todo e na busca por um sorriso em harmonia e uma face equilibrada (Cavalcanti et al., 2017 \& Machado, 2020 \& Miranda, 2020). Rezende et al., (2016), afirma que esses avanços da odontologia, abrangeram um importante viés na valorização de padrões estéticos, pois em alguns casos esses padrões passaram a ser considerados definidores de condições de saúde bucal. Andrade \& Souza (2010), reiteram que a busca pela elevação da autoestima é uma forma de expandir a sua capacidade de ser feliz, uma vez que a maneira como o ser humano se percebe, é manifestada diretamente em suas ações, na vida profissional e pessoal. Portanto, há um consenso entre os autores, no que tange a essa busca por procedimentos estéticos, de que ela está fortemente ligada ao desejo de melhorar a qualidade de vida e consequentemente o bem estar biopsicossocial.

A harmonização orofacial é a especialidade odontológica responsável por tornar os terços da face de um paciente mais proporcionais esteticamente através de procedimentos como: preenchimento facial com biomateriais, aplicação da toxina botulínica, bichectomia, lipoplastia cervical e rinomodelação (Rodrigues, 2021). Os conceitos hoje vigentes para o diagnóstico e plano de tratamento odontológico remetem ao equilíbrio e harmonia dos traços faciais de cada paciente de forma individual, através da análise facial. Esse exame, que por muito tempo, foi utilizado por ortodontistas e cirurgiões bucomaxilofaciais, nos dias de hoje deve estar à disposição de qualquer especialidade odontológica, principalmente as que trabalham com estética (Machado, 2020). De acordo com Miranda (2020), estudos mostram que três fatores são determinantes para uma análise do perfil facial, como: forma, proporcionalidade e simetria. A forma geral da face pode ser descrita qualitativamente de redonda, oval ou quadrada, a análise vertical da face em longa ou curta e também descrita através de uma análise horizontal como larga ou estreita. A face deve ser simétrica em tamanho, forma e arranjo dos componentes faciais. A simetria é avaliada pela comparação de ambos os lados, sendo eles os lados direito e esquerdo da face, tendo como o ponto de referência o plano sagital mediano, como as estruturas que atravessam os pontos médios da glabela, da ponta do nariz, do lábio superior e do mento.

Baseando-se nisso, inúmeros pesquisadores tentaram estabelecer quais eram as características faciais responsáveis pela beleza. Alguns defendiam a ideia de que a estética agradável estaria associada à harmonia e ao equilíbrio entre as partes constituintes do perfil facial (Reis et al., 2011 \& Grejo, 2020). Ao tempo em que outros, observaram que a beleza estaria associada à coincidência das proporções faciais com a proporção áurea (Grejo, 2020) ou que as faces esteticamente mais agradáveis seriam aquelas cujas medidas faciais coincidem com as medidas faciais médias da população a qual pertencem. No entanto, todas essas teorias foram testadas e não foi possível estabelecer uma característica facial responsável pela beleza. Por isso, ao estabelecer um plano de tratamento e ao definir os objetivos, deve-se considerar e esclarecer ao paciente todas as possibilidades e limitações do seu caso, eliminando, dessa forma, expectativas irreais, considerando a possibilidade de tratamento multidisciplinar e a eventual indicação para outros profissionais (Miranda, 2020).

Para entender a crescente busca pela harmonização orofacial por parte desses pacientes, é preciso observar as circunstâncias atuais. Afinal, o que eles desejam? (Cavalcanti et al., 2017). Para que o profissional conheça as expectativas dos seus pacientes sobre o atendimento é indispensável que haja empatia e acolhimento, e pontua essas qualidades que promovem uma assistência humanizada, permitindo que o profissional adapte sua prática às necessidades do paciente, melhorando a qualidade do atendimento e o relacionamento entre ambos (Rezende et al., 2016). Segundo Moura (2021), um sorriso esteticamente agradável é um dos principais desejos da maioria, incentivados pelos padrões estéticos impostos pela sociedade. Mas, para Cavalcanti et al., (2017) os pedidos vão muito além do sorriso, os pacientes buscam saúde, função, beleza, rejuvenescimento, harmonia e bem-estar, ou seja, estão mais exigentes na busca da harmonia entre um belo sorriso e uma face 
equilibrada. Rodrigues et al., (2021) justifica que isso ocorre porque a Odontologia vem tratando o paciente de maneira mais abrangente, indo além do que é padronizado.

Durante sua formação, o cirurgião-dentista tem como objetivo aprender diversas competências e habilidades específicas, entre elas, a de comunicação com o paciente (Canalli et al., 2012). Ou seja, desde a graduação ele deve iniciar a prática do atendimento humanizado e do acolhimento ao paciente, pois é necessário formar profissionais preparados para além da habilidade técnica (Guerra et al., 2010). Nessa abordagem estão implícitos: ouvir, acolher; esclarecer; sanar dúvidas (Kessamiguiemon et al., 2017). No entanto, para Canalli et al., (2012) na prática diária o diálogo profissional-paciente, na maioria das vezes se torna mecanizado e desprovido do real desejo de ouvir atentamente o que o paciente tem a dizer. Para Rezende et al., (2016) desde o primeiro contato entre o profissional e o paciente já é possível estabelecer uma linha de cuidado, que permite a construção do vínculo e da ação terapêutica. Guerra et al., (2010) explica que o atendimento odontológico humanizado pode ser compreendido como uma equipe, formada por pessoas servindo umas às outras em situação de vulnerabilidade e fragilidade, estabelecendo assim, um relacionamento interno de confiança entre o cirurgião-dentista e paciente, bem como, auxiliares, técnicos e todos os envolvidos. Dessa maneira, a confiança, a segurança, a tranquilidade e a serenidade serão sempre reforçadas durante as consultas.

Segundo, Rodrigues (2021), a Odontologia foi conceituada como uma profissão que deve ser exercida em benefício da saúde do ser humano, sem discriminação de qualquer forma ou pretexto. No âmbito odontológico em geral, há direitos e deveres a serem cumpridos. No que diz respeito aos aspectos éticos: Todo cirurgião-dentista tem o direito de diagnosticar, planejar e executar tratamentos segundo sua conviç̧ão e dentro dos limites de suas atribuições (BRASIL, Art $5^{\circ}$ - inciso I; Res. CFO - 118 - CEO, 2012). Porém, no que se refere ao tratamento humanizado, Guerra et al., (2014) defende que é indispensável que o profissional reconheça o direito do paciente de participar, e não apenas ser "informado" das decisões que levam ao delineamento do seu plano de tratamento.

Rodrigues (2021), pontua ainda conforme a Resolução do Conselho Federal de Odontologia (CFO - 118 - CEO, 2012), que o cirurgião-dentista deve zelar e trabalhar pelo perfeito desempenho ético da Odontologia e pelo prestígio e bom conceito da profissão; bem como zelar pela saúde e pela dignidade do paciente; Não devendo envolver-se em prática de atos que impliquem na mercantilização da Odontologia ou sua má conceituação; E na relação profissional-paciente sempre esclarecer adequadamente os propósitos, riscos, custos e alternativas do tratamento, entre outros. Kessamiguiemon et al., (2017) aborda pontos semelhantes e os coloca como práticas voltadas para um atendimento mais humanizado. Ainda segundo o Código de Ética Odontológica (CEO, 2012), o cirurgião-dentista tem direito a renunciar a realizar o atendimento do paciente, quando a critério do profissional, sejam constatados fatos que prejudiquem o bom relacionamento com o paciente ou o pleno desempenho profissional.

Muitas pessoas alimentam uma verdadeira aversão pela própria aparência e devido à baixa autoestima, acabam desencadeando quadros de ansiedade interpessoal e em muitos casos o transtorno da imagem corporal dismórfica (TDC) (Rezende et al., 2016). O Transtorno Dismórfico Corporal consiste numa percepção distorcida da imagem corporal, provocando preocupações absurdas sobre a aparência física. Essa corrida desenfreada pelo belo, pode levar as pessoas a tornarem-se cada vez mais insatisfeitas e deprimidas, desconfortáveis com o próprio corpo e com a autoestima, tudo isso por estar em busca de um "ideal de beleza" (Strehlau et al., 2015).

Silva et al., (2010) destaca a importância da ética no exercício da profissão, sugerindo que o cirurgião-dentista tenha o preparo para lidar com situações onde o desempenho profissional entre em divergência com as expectativas do paciente, fazendo-o entender que estética e beleza são importantes, mas não devem se tornar uma obsessão. 


\section{Protocolo}

O protocolo aqui sugerido tem como objetivo a realização de um atendimento mais humanizado ao paciente:

- Essa abordagem consiste em ouvir, acolher; esclarecer; sanar dúvidas (Kessamiguiemon et al., 2017).

- Toda e qualquer ação médica e/ou odontológica deve ser baseada em seis requisitos fundamentais: ser focada no paciente, segura, eficiente, feita no tempo adequado e possível ao paciente (Cerri et al., 2015).

- $\mathrm{O}$ atendimento deve ser individualizado, com tempo de qualidade, respeito, bioética, humanidade, sempre valorizando o melhor para o ser humano, pois não se deve fazer algo, para que posteriormente seja necessário refazer ou desfazer (Cerri et al., 2015).

- A partir do primeiro contato entre profissional-paciente, deve ser oferecido um ambiente agradável (Cerri et al., 2015), proporcionando assim um acolhimento, deixando-o à vontade para se expor, construindo assim, um vínculo e ação terapêutica (Rezende et al., 2016).

- O profissional deve dar liberdade para o paciente falar e refletir sobre o que o aflige e suas possíveis causas (Rezende et al., 2016).

- Faz-se a escuta, que é o momento para esclarecimento, que permite aumentar o arsenal de informações sobre ele (Rezende et al., 2016).

- O cirurgião-dentista deve procurar compreender as pessoas que o procuram, pois isso gera uma relação de empatia que torna o paciente mais colaborador com o tratamento (Guerra et al., 2014).

- O prontuário deve ser feito de forma adequada e minuciosa. (Amorim et al., 2016). Enfocando a particularidade de cada paciente, que o profissional venha a atender (Cerri et al., 2015).

- No diagnóstico, o profissional deve observar as características faciais do paciente. (Reis et al., 2011). Através da analise facial, que pode ser: de maneira subjetiva, onde serão feitas marcações de pontos, linhas e ângulos, sendo possível uma comparação com referências do padrão de normalidade, e de maneira objetiva que será feita durante a avaliação, com exame clínico e fotográfico (Grejo, 2020).

- Os aspectos étnicos, culturais e individuais do paciente devem ser considerados. Os aspectos que o agradam devem ser mantidos, e quando possível, acentuados com o tratamento. Respeitando e tentando utilizar os mesmos parâmetros de avaliação estética do próprio paciente. (Reis et al., 2011).

- O paciente deve ser informado sobre as possibilidades de tratamento para o seu caso, ele precisa ser esclarecido sobre todos os procedimentos, com descrição dos materiais que serão utilizados e a região envolvida (Amorim et al., 2016).

- Durante a elaboração e execução do plano de tratamento, cada paciente deve ter a sua particularidade respeitada, sendo feito com atenção, planejamento e execução, diferentes dos demais pacientes (Cerri et al., 2015).

- Por fim, em tudo o que for fazer, o cirurgião-dentista deve sobretudo prezar pelo bom senso, zelar e trabalhar pelo perfeito desempenho ético da Odontologia e pelo prestígio e bom conceito da profissão, evitando a prática de atos que visem a mercantilização da Odontologia ou sua má conceituação (Rodrigues, 2021).

\section{Conclusão}

- Nesta revisão integrativa foi possível perceber que a humanização vem sendo exaltada na legislação como essencial no processo de formação do cirurgião-dentista, e as ações de acolhimento na Odontologia podem ser consideradas fundamentais para melhoria da qualidade de assistência prestada. 
- Nesse caso, é imprescindível que o cirurgião-dentista desenvolva práticas e habilidades profissionais, estando apto a oferecer saúde, através da harmonia dentária e facial, visto que cada vez mais vem aumentando essa procura, em decorrência da valorização do rosto e do sorriso na vida das pessoas.

- Portanto, a saúde está totalmente ligada aos cuidados estéticos, e é, sobretudo uma responsabilidade promovê-la, bem como, um ato de amor, que é intrínseco do ser humano.

- Por fim, sugere-se o desenvolvimento de novas pesquisas relacionadas ao tema, principalmente estudos de campo, a fim de analisar sobre a aplicabilidade e adesão dos profissionais diante do acesso as informações acerca do assunto propagado.

\section{Referencia}

Andrade, E. R. D., \& Souza, E. R. D. (2010). Autoestima como expressão de saúde mental e dispositivo de mudanças na cultura organizacional da polícia. Psicologia Clínica, 22, 179-195. https://www.scielo.br/j/pc/a/7nn34JCRGyVSYssHqmgjLMs/?format=pdf\&lang=pt

Avelar, C. F. P. D., \& Veiga, R. T. (2013). Como entender a vaidade feminina utilizando a autoestima e a personalidade. Revista de Administração de Empresas, 53, 338-349. https://doi.org/10.1590/S0034-75902013000400002

Borba, T. J., \& Thives, F. M. (2011). Uma reflexão sobre a influência da estética na autoestima, auto-motivação e bem estar do ser humano. Tcc (graduação) Curso de Cosmetologia e Estética, Universidade do Vale do Itajaí Balneário Camboriú. http://siaibib01.univali.br/pdf/Tamila\%20Josiane\%20Borba.pdf

Canalli, C. D. S. E., Gonçalves, S. S., Chevitarese, L., da Gama Silveira, R., \& Miasato, J. M. (2011). A humanização na Odontologia: uma reflexão sobre a prática educativa. Revista Brasileira de Odontologia, 68(1), 44. http://dx.doi.org/10.18363/rbo.v68n1.p.44=

Canalli, C. D. S. E., Silveira, R. D. G., Miasato, J. M., \& Chevitarese, L. (2012). Humanização na relação cirurgião-dentista-paciente. Rev. odontol. Univ. Cid. São Paulo (Online). https://arquivos.cruzeirodosuleducacional.edu.br/principal/old/revista_odontologia/pdf/setembro_dezembro_2012/unicid_24_03_2205.pdf

Cavalcanti, A. N., Azevedo, J. F., \& Mathias, P. (2017). Harmonização Orofacial: a Odontologia além do sorriso. Journal of Dentistry \& Public Health, 8(2), 35-36. https://www5.bahiana.edu.br/index.php/odontologia/article/download/1454/1085

Cerri, A., Guarim, J. D. A., \& Genovese, W. J. (2015). Planejamento e diagnóstico em Odontologia com os princípios bioéticos. Revista da Associacao Paulista de Cirurgioes Dentistas, 69(3), 216-225. http://revodonto.bvsalud.org/scielo.php?pid=S0004-52762015000200003\&script=sci_arttext\&tlng=pt

Conselho Federal de Odontologia. (2012). 2012 Resolução Nº 118. ttps://website.cfo.org.br/wp-content/uploads/2018/03/codigo_etica.pdf

de Lima Amorim, H. P., Marmol, S. L. P., Cerqueria, S. N. N., da Silva, M. L. C. A., \& da Silva, U. A. (2016). A importância do preenchimento adequado dos prontuários para evitar processos em Odontologia. Arquivos em Odontologia, 52(1). https://periodicos.ufmg.br/inde x.php/arquivosemodontologia/article/view/3686

Garbin, A. J. I., Wakayama, B., Saliba, T. A., \& Garbin, C. A. S. (2019). Harmonização orofacial e suas implicações na odontologia. Braz. J. Surg. Clin. Res, 27(2), 116-122. https://www.mastereditora.com.br/periodico/20190704_103726.pdf

Grejo, C.L.A. (2020). Padrão facial na harmonização. Tese (Especialização em Harmonização Orofacial). Faculdade FACSETE. Guarulhos. São Paulo. http://www.ciodonto.edu.br/monografia/files/original/e529056c597194dc6fb6ffce56701fc2.pdf

Guerra, C. T., de Magalhães Bertoz, A. P., Fajardo, R. S., \& Rezende, M. C. R. A. (2014). Reflexões sobre o conceito de atendimento humanizado em Odontologia. Archives of Health Investigation, 3(6). https://www.archhealthinvestigation.com.br/ArcHI/article/view/72

Junior, R. M., Ribeiro, P. D., Condezo, A. F. B., Cini, M. A., De Antoni, C. C., \& Moreira, R. (2018). Fundamentos da análise facial para harmonização estética na odontologia brasileira. Clínica e Pesquisa em Odontologia-UNITAU, 9(1), 59-65. http://webcache.googleusercontent.com/s earch?q=cache:Vfd0RQpJAngJ:periodicos.unitau.br/ojs/index.php/clipeodonto/article/view/2607+\&cd=1\&hl=pt-BR\&ct=clnk\&gl=br

Kessamiguiemon, V. G. G., Oliveira, K. D. C., \& Brum, S. C. (2017). TEA-Atendimento odontológico: relato de caso. Revista Pró-univerSUS, 8(2), 67-71. http://editora.universidadedevassouras.edu.br/index.php/RPU/article/view/1173

Luiza Fernandes Duarte, A. N. A., \& Cardoso Lírio, F. E. R. N. A. D. A. (2020). Harmonização Orofacial: Revisão de literatura. http://dspace.uniube.br:8080 /jspui/handle/123456789/1374

Machado, L. L. (2020). Atuação do cirurgião dentista na harmonização orofacial. http://hdl.handle.net/10183/214031

Miranda, I. C. Harmonização Facial: O sorriso do exterior para o interior. http://faculdadefacsete.edu.br/monografia/files/ original/d07a3aef8c75a7ac7b8bad44adbdaeb4.pdf

Moura, S.V. (2021). Análise do sorriso com ênfase na posição labial superior visando a reabilitação implantossuportada. Tese (Especialização em Implantodontia). Faculdade FACSETE. https://faculdadefacsete.edu.br/monografia/items/show/3384

Reis, S. A. B., Abrão, J., Claro, C. A. D. A., \& Capelozza Filho, L. (2011). Avaliação dos fatores determinantes da estética do perfil facial. Dental Press Journal of Orthodontics, 16, 57-67. Disponível em: https://doi.org/10.1590/S2176-94512011000100010 
Research, Society and Development, v. 10, n. 14, e173101421709, 2021

(CC BY 4.0) | ISSN 2525-3409 | DOI: http://dx.doi.org/10.33448/rsd-v10i14.21709

Rezende, M. C. R. A., \& Fajardo, R. S. (2016). Abordagem estética na Odontologia. Archives of Health Investigation, 5(1). https://doi.org/10.21270/archi.v5i1.1298

Rezende, M. C. R. A., Lopes, M. R. A. N. E., de Assis Gonçalves, D., Zavanelli, A. C., \& Fajardo, R. S. (2015). Acolhimento e bem estar no atendimento odontológico humanizado: o papel da empatia. Archives of Health Investigation, 4(3). https://www.archhealthinvestigation.com.br/ArcHI/article/view/904

Rodrigues, L. G. (2021). Harmonização orofacial: análise do conhecimento dos Cirurgiões-Dentistas sobre os riscos clínicos e aspectos legais e éticos na prática da rinomodelação e bichectomia. https://repositorio.bc.ufg.br/tede/handle/tede/11264

Schmidt, L. L. (2021). A importância do conhecimento anatômico na realização de procedimentos injetáveis com propósito de harmonização facial. https://repositorio.animaeducacao.com.br/handle/ANIMA/17184

Silva, M. B., Carlini, T. C. B, Gallas, J. C. (2010). A busca excessiva pela beleza. Bauneário Camboriú. Santa Catarina. http://siaibib01.univali.br/pdf/Marcia\%20da\%20Silva,\%20Thuanny\%20Carlini.pdf

Sociedade Brasileira de Cirurgia Plástica-SBCP. (2018). 2018 Pesquisa - Senso. Grafico: Procedimentos cirurgicos e não cirurgicos, pág. 12. Adans Estatística e consultoria. http://www2.cirurgiaplastica.org.br/wp-content/uploads/2019/08/Apresentac\%CC\%A7a\%CC\%83o-Censo-2018_V3.pdf

Strehlau, V. I., Claro, D. P., \& Laban, S. A. (2015). A vaidade impulsiona o consumo de cosméticos e de procedimentos estéticos cirúrgicos nas mulheres? Uma investigação exploratória. Revista de Administração (São Paulo), 50, 73-88. https://doi.org/10.5700/rausp1185 